\title{
The life-cycle of artificial contexts
}

\section{Cycle de vie des contextes artificiels}

\author{
Juan Carlos Augusto ${ }^{1}$, Thomas E. Dickins ${ }^{2}$ \\ ${ }^{1}$ Department of Computer Science, Middlesex University, London UK, J.Augusto@mdx.ac.uk \\ 2 Department of Psychology, Middlesex University, London UK, T.Dickins@mdx.ac.uk
}

\begin{abstract}
Little has been published on connecting contexts as they are handled in natural systems and in computer science. This article selected some natural contexts and use them as an inspiration to highlight possible improvements on how contexts are designed and used in computer science. The authors found there are beneficial lessons and inspirations which have the potential to bring practical benefits as a result of this process.

RESUME. II y a peu de publications concernant des connexions sur la manière dont les systèmes naturels et l'informatique utilisent le contexte. Cet article présente une sélection de contextes naturels qui pourrait fournir une inspiration pour améliorer la conception et l'utilisation des contextes en informatique. Les auteurs pensent qu'il existe des leçons bénéfiques qui ont le potentiel d'apporter des avantages pratiques à la suite de ce processus.

KEYWORDS. Contexts, context-awareness, natural systems, computing systems.

MOTS-CLES. Contextes, sensible au contexte, systèmes naturels, logiciels.
\end{abstract}

\section{Introduction}

Within Computer Science context and context-awareness explain how computing systems are capable of automating tasks in certain subtle problem-solving scenarios. An important early publication from McCarthy [15] led to more detailed Artificial Intelligence studies and at the end of the $20^{\text {th }}$ century a systems engineering view of contexts and context-awareness gained popularity [6]. AI and systems engineering approaches developed independently of one other which has possibly been to the detriment of understanding [2].

Intuitively we are interested in context-aware computing systems as a (simplified) metaphor for context-aware organisms that modify decision-making, and actions based on what they experience and have learnt from real-world engagements. These mimicking systems can be represented by the following Figure 1.1.

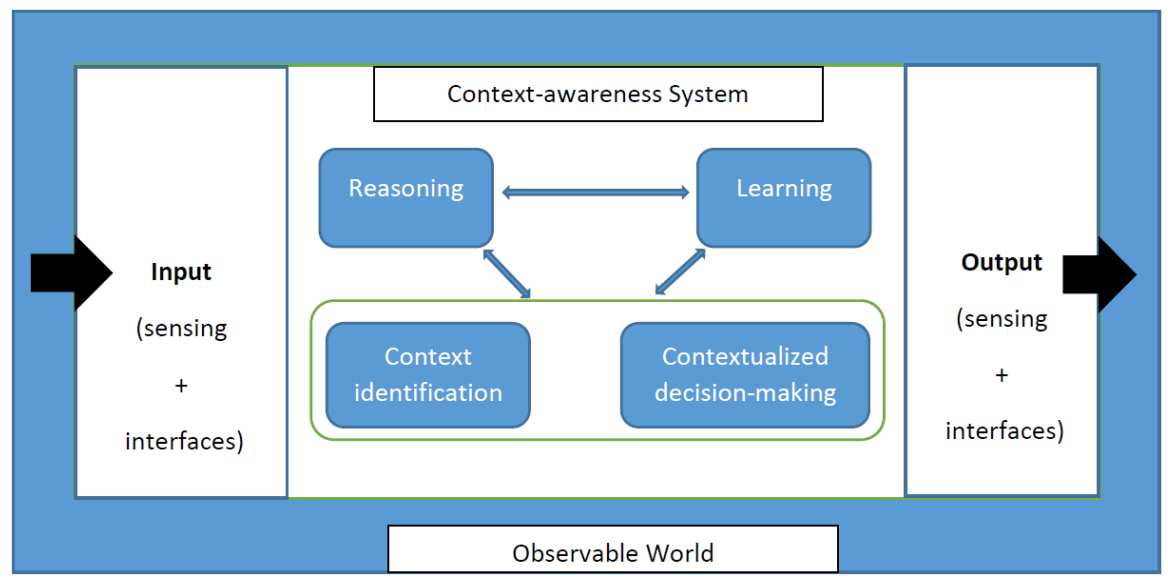

Fig. 1.1. Generic Context-aware System Concept

For clarity we consider sensors as devices which can transform a physical dimension into a digital and actuators as devices which can transform a digital dimension into a physical one. Interfaces allow humans to provide/receive data to/from the system. Internal modules such as 
Reasoning and Learning, Context Identification and Context-based Decision Making are to be considered as typically explored in the areas of Artificial Intelligence or Ubiquitous Computing during the last two decades [1].

\section{Research Focus}

The most consistent attempt to understand context from an interdisciplinary perspective has been conducted by the CONTEXT series of conferences over the last twenty years [see for example, 10]. This community has investigated various perspectives on context in connection with other disciplines inside computer science (e.g., systems modeling and cognitive science) and to some extent to other areas of knowledge outside computing (e.g., philosophy and linguistics). So far little connection has been established with how nature handles context and so there has been no contact with biology. Below we outline some comparisons between how context is managed in computing and in nature. Our hope is to provide some inspiration for the CS community to further develop the concept within the field.

\section{Research Methods}

We have developed a comparative verbal argument based on possible connections between the way that systems in nature handle contexts and the way contexts are used or can be used in computer science and in technological innovation. The comparisons have been organised under four themes: how contexts appear, how they are recognized, how they are redefined, and how they interrelate. Some general lessons have then been drawn.

A guiding framework from biology was that of autopoiesis [11]. Systems (organisms) are considered alive when they have sufficient internal complexity to gain some degree of autonomy from the external world. Autonomy is gained by tracking data in the external environment and that data is used to cause state changes in specific internal systems that render those inputs (or data) informational. Thus, data has a role within a specific system or context [9]. We regard the existence of such systems the result of evolution.

\section{Research findings/results}

So far we have established connections of interest on a number of dimensions, including the differences and parallels in each area on: the motivation for contexts consideration, their use, their adaptation through time, the drivers of how systems react to contexts.

\subsection{How Contexts Appear?}

In nature: Evolution by natural selection is a statistical outcome of variation, differential reproductive success, and inheritance. As traits are heritable more competitive trait variants come to dominate in future populations, while less competitive variants reduce in relative frequency. In its general form, this Darwinian process can happen in any system that permits these three components but evolutionary biology focuses upon genetic variation, leading to variation in expressed traits [22]. Thus, reproductively successful traits cause their underlying genes to increase in relative frequency within the population. These traits are adaptations. Genes also play a coding role in development as they carry sequentially encoded data within DNA that informs protein synthesis and related activities. The genotype of an individual is a dataset that delivers constraints for building traits, and those constraints have been selected for during evolution. In this way a genotype represents responses to past contexts. 
Natural selection is a gradual process. Environmental change therefore presents a problem if it is too fast for selection to respond to. This threatens the genotype of an organism, and the genotype therefore require robustness solutions. We give four: Conservative bet hedging is where genotypes build generalist traits that do relatively well in many contexts. In diversified bet hedging a genotype produces, for example, two versions of a phenotype that do well in version-specific contexts. Those versions are produced on a probability distribution that matches the likelihood of encountering those contexts [20]. Developmental plasticity results from organisms being sensitive to data early in ontogeny. That data will predict the likely future environment and set the development of the organism on an appropriate future context sensitive trajectory. For example, if the organism is born into a low food environment, that provides key nutritional data about likely food resource in the future. This data will cause the organism to invest what nutrition there is into building essential organs (brains, reproductive mechanisms) at the expense of growth elsewhere [16]. Physiological plasticity includes multiple physiological response systems, including behavioural responding, to day-to-day, and moment-to-moment data. These allow organisms to do things such as learn in changeable environments, but all such mechanisms are delimited. For example, associative learning cannot simply be a question of associating everything because nothing would be learnt. Learning mechanisms are directed at particular contexts, and those parameters are a function of natural selection [7].

In computing: Often within computing systems engineering, contexts are rigidly set by developers based on the services expected and the resources available. If light automation is perceived as useful or necessary in a room then hardware (e.g., movement sensors and actuating lights) and software (which implements an algorithm to turn lights on/off based on the sensor detecting movement) is acquired or created to address that need. Contexts are created primarily by the developer, who is aiming to solve a specific problem. In nature new variants are produced at random with respect to the selected outcome and when by chance they facilitate differential success they are retained. Developers more commonly design new variants based on hypothesis and theory.

\subsection{How are Contexts Recognized?}

In nature: A standard heuristic in evolutionary biology is to think of natural selection as a design process. Thus, traits are modelled as optimal solutions to environmental problems, where optimality is understood in terms of fitness maximization. Fitness is usually understood in terms of the sum of direct and indirect reproductive success, and is captured by inclusive fitness theory [23]. Given this, sensory systems are to be seen as designed to take specific kinds of input from the environment. Here we regard environment as both internal and external to the organism depending upon the task demands. Typical examples of sensory systems will include visual, auditory, olfactory, haptic, and gustatory input systems. But we can reduce our focus to draw out a generality about biological mechanisms as systems of constraints upon the flow of free (available) energy [4]. For such mechanisms to operate they rely upon an input that tips the energetic dynamics into a particular pathway, and the mechanism has been designed by selection to respond only to specific sources, or kinds of input. This response is determined by physical properties of the mechanism, for example membrane properties of the cell or the availability of binding sites for an enzyme. In the presence of the right input those properties will facilitate responses and onward work. An input is labelled as right with reference to the selection history of the focal mechanism.

In computing: Sensors are excited to a fixed threshold, but sometimes developers modify thresholds. Actuation can also modify intensity of outcome for example, sometimes only on/off outcomes are available and at others a range of output values are available (e.g., dimmable lights or temperature gradations). The representation of input and output values can vary depending on the various programming strategies (Rule-based, Fuzzy Logics, Artificial Neural Networks, etc.) used to process them [18]. 


\subsection{How are Contexts Redefined?}

In nature: Over an evolutionary timescale selection can shift a population from exploiting one environmental context to exploiting another. During a lifespan developmental and physiological responses can achieve shifts in context. For example, think of an animal that can eat two types of food, A and B. Type A has higher calorie returns than B. We should expect the animal to prefer A, and to reject $\mathrm{B}$ in favour of $\mathrm{A}$ when $\mathrm{A}$ is abundant. But if the relative abundance of $\mathrm{A}$ falls below a certain threshold we should expect the animal to starting choosing B also. Thus, the animal will shift from a specialist (A only) to generalist (A \& B) foraging strategy due to exposures and sensitivity to abundance (assayed via encounter rate). This is an example of an animal changing its response to environmental data, and effectively altering its foraging context [5].

In computing: Operational conditions of contexts are initially forecast at the time of design, then developed and deployed. Modification of design assumptions may lead to error within contexts where previously the system had worked. This will lead to further modification. This process is not unlike natural selection in terms of its extended time period and a requirement to redesign the system. Some systems have a small degree of flexibility due to automated learning strategies (supervised, semi-supervised or unsupervised) that are encoded in the representation model of choice (rule-based, fuzzy logics, artificial neural networks, etc.). This too is analogous to the plasticity responses found in nature.

\subsection{How Contexts Inter-relate?}

In nature: Biological models can be described as local hierarchies of related mechanisms, and thus related contexts. Each mechanism provides a context for inputs, those inputs change the state of the mechanism and those state changes can cause outputs which act as inputs to higher level mechanisms. Overall, mechanisms can find use in more than one local hierarchy and are best described as involved in a heterarchy [8]. These networks are effectively cooperative. Cooperation between cells is the hallmark of multicellular organisms and regarded as a major transition in evolutionary history [14].

In computing: Computing systems are similarly organized either as hierarchies or as networks of interconnected concepts. Whatever their organization, again the prevalent model is that the developer sets a given organization and that is developed and deployed and very much remains the same unless performance issues trigger a revision, however these are rare. Systems that work are rarely changed and if changes are applied they tend to be minimal to solve a specific malfunction.

\subsection{Some Nature Inspired Lessons for Context Design}

Based on the considerations above, we can envision some directions for the engineering of context-aware systems where more flexibility is embedded in the system for contexts to be created (section 4.1) identified (section 4.2), redefined (section 4.3) and related to each other (section 4.4).

Creation: much of the state-of-the-art in computer science can be characterized as rigid in that it pre-specifies contexts without acknowledging possible change. Design requires a focus upon robustness solutions as in nature. A possibility would be to direct machine learning to discover contexts and track contextual change. For example, if the act of a person getting up from bed and going to the bathroom is associated that person to turn on lights in the corridor and the bathroom, the system can detect that pattern through time and use it to anticipate and automate lighting [3]. In Figure 4.1 we represent the unfolding of the conditions announcing the situation with a sequence of events $e_{1} \ldots e_{n}$ and the culminating event of interest with $e$. We represent with $d_{1} \ldots d_{n}$ the historical data that has been learnt to be associated with outcome $k$ ' which can be combined with other pieces of knowledge $k_{1} \ldots k_{m}$ to provide an outcome $O$. 


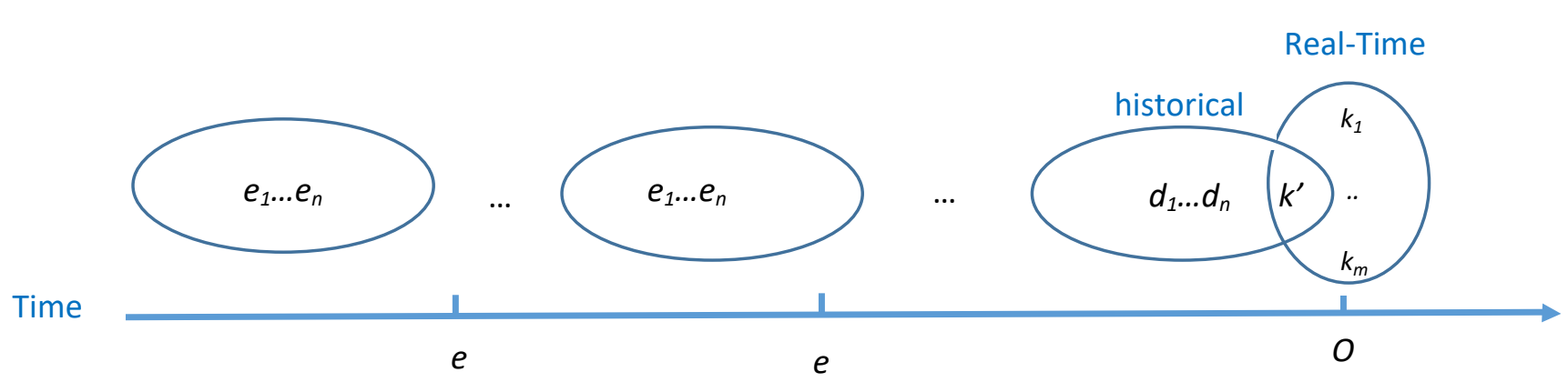

Fig. 4.1. Identifying historical patterns

Identification: if some of those pieces of knowledge are missing (either $k^{\prime}$ or any of the $k_{1} \ldots k_{m}$ ) then the context is not identified, the system does not act on it, and it does not produce the outcome. However, these Boolean takes on context identification can also be made fuzzy and investigate what complex combinations of circumstances can relate to a context being unfolding or having been reached, and in which levels of expression these need to reach to satisfy context conditions. Such fuzziness could be dealt with by probability distributions, in something equivalent to bet hedging strategies in nature. More generally, in dynamic contexts prior probability structures associated with $e$ to $O$ transitions are updated. This Bayesian learning is possibly analogous to aspects of natural selection where the genome is updated after novel environmental exposure assuming available genetic variation [17].

Redefinition: contexts can be dynamically redefined, the conditions which characterize a context $e_{1} \ldots e_{i}$ can be updated to $e_{1} \ldots e_{j}$ and to $e_{1} \ldots e_{k}$ when $e_{j}$ and $e_{k}$ are found to be relevant (Figure 4.2). This happens in nature when organs and organisms adapt to changing circumstances, however adaptation in nature is usually noticeable after considerable time (for example, a Pavlov's reaction may disappear if the animal unlearns the association with the stimuli) [19]. In artificial context-aware systems it is feasible to do a quicker adaptation, there are motivations to do it more often and quickly, however this is not currently the state of the art where systems are more hardwired and permanent and although it will be easier to achieve it the community has focused on more fundamental shortcomings. Most research in relation to adaptation and context-awareness refer to applications which are adaptive thanks to the use of (rigid) context-awareness, not to contextawareness that it is itself flexibly renewed through adaptation.

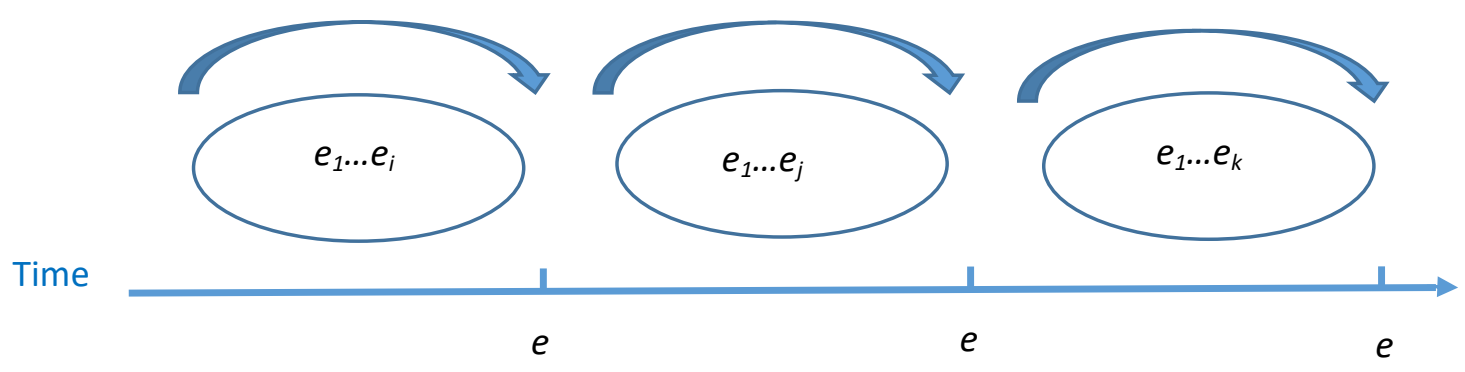

Fig. 4.2. Adaptive Learning of contexts

In evolutionary terms entities may be encouraged to develop in specific directions based on the feedback they receive from the environment (e.g., changing food acquisition strategies to be rewarded with a better energy intake) [21]. This is another area which can do with more attention within the context-aware community: system feedback, for example through techniques such as Reinforcement Learning where a feature $f$ is suggested to the system as missing until it is incorporated (Figure 4.3). 


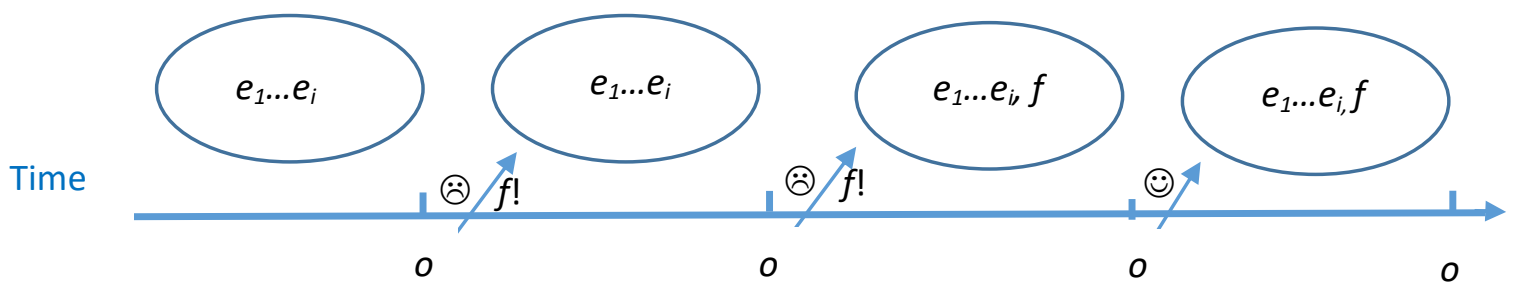

Fig. 4.3. Context-aware systems can benefit with feedback

Inter-relatedness: the inter-relation between contexts is also under researched in computing. Typically, there is a relation between contexts in designed systems, but it is established during the design process and fixed. System revisions due to extrinsic changes in task demands, for example, require expensive revisions with no guarantee of outcome. In general revisions are avoided where possible. In nature the addition of systems to organisms is a consequence of natural selection. But it is most commonly selection on regulatory genes that organize how specific compartmentalized modules are related to one another [12]. This permits evolvability as it prevents new genetic variation destabilizing the whole, whilst allowing novelty in response to new contexts. In short, the contexts that have changed can be dealt with but not at the expense of other systems for unchanged contexts. A common example of this modularity is that of Hox genes that regulate the basic bilateral body plan across multiple taxa [13]. Reorganizing where Hox genes are expressed determines where body parts such as legs and antennae are placed in development.

The flexibilities discussed above will lead to context-aware systems which are more flexible and self-sufficient. This is far from the state of the art and it will represent a new generation of contextaware systems.

\section{Research Conclusion}

We believe there is a benefit on more closely investigating the interconnections of these two seemingly disconnected areas and using the main characteristics of strategies perfected in nature to inspire innovative context-based system design by system developers. We looked at how contexts are generated and recognized, how they change and inter-relate. All processes which happen in nature and are also important in computing. We illustrated how these processes in nature may lead to reflection on these within computing and hopefully to future progress at a theoretical and then practical level.

\section{References}

[1] Alegre. U., Augusto, J. C., Clark, T. (2016): Engineering context-aware systems and applications: A survey. Journal of Systems and Software. 117: 55-83. Elsevier.

[2] Augusto, J. C., Aztiria, A., Kramer, D., Alegre U. (2017) A Survey on the Evolution of the Notion of ContextAwareness. Applied Artificial Intelligence 31(7-8): 613-642. Taylor and Francis.

[3] Aztiria, A., Augusto, J. C., Basagoiti, R., Izaguirre, A., and Cook, D. J. (2013) Learning frequent behaviours of the users in Intelligent Environments. IEEE's Transactions on Systems, Man and Cybernetics: Systems 43(6):12651278. IEEE Press.

[4] Bechtel W, Bich L. 2021 Grounding cognition: heterarchical control mechanisms in biology. Philos. Trans. R. Soc. B Biol. Sci. 376, 20190751. (doi:10.1098/rstb.2019.0751)

[5] Charnov EL. 1976 Optimal Foraging : Attack Strategy of a Mantid. Am. Nat. 110, 141-151.

[6] Dey, A. K. and G. D. Abowd (1999). Towards a Better Understanding of Context and Context-Awareness. Computing Systems 40 (3), 304-307. 
[7] Dingemanse NJ, Kazem AJN, Réale D, Wright J. 2010 Behavioural reaction norms: animal personality meets individual plasticity. Trends Ecol. Evol. 25, 81-89. (doi:10.1016/j.tree.2009.07.013)

[8] Eronen MI. 2015 Levels of organization: a deflationary account. Biol. Philos. 30, 39-58.

[9] Floridi L. 2010 Information: A Very Short Introduction. Oxford: Oxford University Press.

[10] Gabor B. and Bouquet P. (2019) Modeling and Using Context. Proceedings of the 11th International and Interdisciplinary Conference (CONTEXT 2019). Gabor B. and Bouquet P. (Eds.). Trento, Italy.

[11] Gershenson C. 2015 Requisite variety, autopoiesis, and self-organization. Kybernetes 44, 866-873.

[12] Kirschner MW, Gerhart JC. 2010 Facilitated Variation. In Evolution: The Extended Synthesis (eds M Pigliucci, GB Müller), pp. 253-280. Cambridge MA: MIT Press.

[13] Mallo M, Wellik DM, Deschamps J. 2010 Hox genes and regional patterning of the vertebrate body plan. Dev. Biol. 344, 7-15. (doi:10.1016/j.ydbio.2010.04.024)

[14] Maynard-Smith J, Szathmary E. 1995 The Major Transitions in Evolution. Oxford: Oxford University Press.

[15] McCarthy, J. (1993). Notes on Formalizing Context. In Proceedings of the Thirteenth IJCAI.

[16] Nettle D, Bateson M. 2015 Adaptive developmental plasticity: what is it, how can we recognize it and when can it evolve? Proc R Soc B 282, 20151005-. (doi:10.1098/rspb.2015.1005)

[17] Richerson PJ. 2018 An integrated bayesian theory of phenotypic flexibility. Behav. Processes 161, 54-64.

[18] Russell, S. J., and Norvig, P.: Artificial Intelligence - A Modern Approach, Third International Edition. Pearson Education 2010, ISBN 978-0-13-207148-2, pp. I-XVIII, 1-1132

[19] Staddon, J. E. R. (2016). Adaptive Behavior and Learning (Second). Cambridge University Press.

[20] Starrfelt J, Kokko H. 2012 Bet-hedging-a triple trade-off between means, variances and correlations. Biol. Rev. 87, 742-755. (doi:10.1111/j.1469-185X.2012.00225.x)

[21] Stephens, D. W., Brown, J. S., \& Ydenberg, R. C. (2007). Foraging: Behavior and Ecology.

[22] Webb RH. 2011 If evolution is the answer, what is the question? J. Evol. Psychol. 9, 91-107.

[23] West SA, Gardner A. 2013 Adaptation and Inclusive Fitness. Curr. Biol. 23, R577-R584. 